\title{
Role of bacteria in acute exacerbations of chronic obstructive pulmonary disease
}

\section{Levent Erkan \\ Oguz Uzun \\ Serhat Findik \\ Didem Katar \\ Ahmet Sanic \\ Atilla G Atici}

Ondokuz Mayis University, Faculty of Medicine, Department of Pulmonary Medicine, Samsun, Turkey
Correspondence: Serhat Findik

Ondokuz Mayis University, Faculty of Medicine, Department of Pulmonary Medicine, TR : 55139, Kurupelit,

Samsun, Turkey

Tel +903623121919 ex. 3124

Fax +90362 457604 I

Email serhatfindikI@yahoo.com
Background and study objective: Infections are major causes of acute exacerbations of chronic obstructive pulmonary disease (COPD) which result in significant mortality and morbidity. The primary aim of the study was to determine the microbiological spectrum including atypical agents in acute exacerbations. The secondary aim was to evaluate resistance patterns in the microorganisms.

Methods: The sputum culture of 75 patients admitted to our clinic from January 1, 1999 to December 31, 2002 was evaluated prospectively, for aerobic Gram-positive and Gram-negative bacteria, and serologically for Chlamydophila pneumoniae and Mycoplasma pneumoniae. Sensitivity patterns in potentially pathogenic microorganisms (PPMs) were also investigated.

Results: An infectious agent was identified in 46 patients, either serologically or with sputum culture. Pathogens most commonly demonstrated were: Haemophilus influenzae (30\%), Chlamydophila pneumoniae (17\%), and Mycoplasma pneumoniae (9\%). Mixed infections were diagnosed in 9 patients. PPMs showed a high resistance rate to commonly used antibiotics.

Conclusion: We have shown that microorganisms causing acute exacerbations of COPD are not only typical bacteria (46\%) but also atypical pathogens (26\%), with unpredictable high rates. Typical agents showed a high resistance to commonly used antibiotics.

Keywords: chronic obstructive pulmonary disease, acute exacerbation, infection, atypical pathogens, Haemophilus influenzae

\section{Introduction}

Chronic obstructive pulmonary disease (COPD) is one of the most prevalent disease in the world. Furthermore, the number of individuals affected has grown since the 1980s and this increase is expected to continue during the next 20 years (GOLD 2001). COPD is characterized by intermittent acute exacerbations associated with worsening symptoms and lung function. These acute exacerbations contribute considerably to mortality and diminished quality of life (Seemungal et al 1998).

Several etiologic factors alone or in combination may cause exacerbations of COPD. In $50 \%-70 \%$ of acute exacerbations of COPD, the pathophysiological basis is usually infectious (Ball 1995). Haemophilus influenzae is the most frequent bacterium isolated in all series followed by Streptococcus pneumoniae, and Moraxella catarrhalis, and Pseudomonas aeruginosa (Ball 1995; Monso et al 1995; Soler et al 1998; Miravitlles et al 1999). Some reports on the role of Chlamydophila pneumoniae and Mycoplasma pneumoniae in acute exacerbations of COPD have appeared in recent years (Mogulkoc et al 1999; Lieberman et al 2001), showing that they are important causes of community-acquired pneumonia. However, these bacteria are usually almost totally absent from discussion on acute exacerbations of COPD. In the light of this knowledge, we prospectively designed a study to disclose the role of bacteria including Ch. pneumoniae and M. pneumoniae and sensitivity patterns of PPMs in acute exacerbations of COPD. 


\section{Material and methods}

Patient selection

All patients hospitalized with acute exacerbation of COPD in our clinic between January 1, 1999 and December 31, 2002 were prospectively evaluated. All patients were diagnosed as having COPD, according to the criteria of the American Thoracic Society (ATS 1995). Acute exacerbation was defined as the presence of an increase in at least two of the three following symptoms: dyspnea, cough and sputum production.

Admission to hospital was according to the clinical situation of the patient, or the presence of complicating factors such as respiratory failure by a senior chest physician, experienced in the management of COPD. Exclusion criteria were: (1) outpatient status; (2) treatment with any antibiotic within $48 \mathrm{~h}$ before admission; (3) absence of an adequate sputum specimen as determined by Gram stain; (4) evidence of bronchiectasis and/or pneumonia; (5) malignancy or severe immunosupression; and (6) the need for mechanical ventilation. After a thorough history and physical examination, chest radiography and pulmonary function tests were performed. Heamatologic, microbiologic, and serologic investigations were done on the same day. Serologic investigation for Ch. pneumoniae and M. pneumoniae was repeated 21 days later.

\section{Laboratory investigations}

\section{Sputum cultures}

At least one sample of spontaneously expectorated sputum for microbiological evaluation was obtained from all patients during admission. A Gram's stain of sputum in the area of maximal purulence was examined for polymorphonuclear leukocytes and epitelial cells. The criteria used to determine whether a sputum sample was acceptable for analysis were: a microbiological study using a low-magnification field $(\times 100)$ revealed $<10$ epithelial cells and $>25$ leukocytes (Murray et al 1975). Sputum specimens not fullfilling these criteria were not cultured because they were not considered representative bronchial samples.

Sputum samples were plated on blood, eosin-methylene blue and chocolate agar and incubated at $37^{\circ} \mathrm{C}$ for both $24 \mathrm{~h}$ and $48 \mathrm{~h}$. All microorganisms isolated were identified through standard laboratory methods (Isenberg et al 1991).

Bacterial agents were classified into PPM or non-PPMs, as described by Cabello and colleagues (1997). PPMs were regarded as significant only if they achieved $>10^{6} \mathrm{cfu}$, except for $S$. pneumoniae where $10^{5} \mathrm{cfu}$ was deemed sufficent. A PPM had to grow in significant concentrations irrespective of presence of non-PPMs to be considered a potential causative agent of an exacerbation.

\section{Serology}

All sera were investigated for the presence of immunoglobulin $\mathrm{G}(\mathrm{IgG})$ and immunoglobulin M (IgM) antibodies to Chlamydophila species, using indirect-microimmunofluorescence test (Vircell SL, Microgen Bioproducts Inc., Camberley, UK). Serum antibodies against $C h$. pneumoniae elementary bodies were detected with fluorescein-conjugated monoclonal goat antihuman Ig-subclass antibodies. An isolated IgM titer of $\geq 1: 16$, or $\operatorname{IgG}$ titer of $\geq 1: 512$, or a fourfold increase in titer of $\operatorname{IgG}$ or IgM, was considered evidence of acute Ch. pneumoniae infection. Past Ch. pneumoniae infection (chronic or preexisting antibody) was defined as an IgG titer of 1:64 to $1: 256$.

The presence of circulating IgG and IgM antibodies to $M$. pneumoniae was detected with an indirectmicroimmunofluorescence test (Zeus Scientific, Inc., NJ, USA). A 4-fold change in titer of $\operatorname{IgG}$ or IgM, or an isolated IgG titer of $\geq 1: 128$ or IgM titer of $\geq 1: 16$, was considered to evidence of acute infection.

\section{Statistical analysis}

Statistical analysis was performed with a statistical software system (SPSS, version 10.0; SPSS Inc., Chicago, IL, USA). Student's t-test and chi-square tests were used ( $\mathrm{p}$ value of $\leq 0.05$ was considered statistically significant).

\section{Results}

The study included 75 patients who had been diagnosed with acute exacerbation of COPD from a total of 156 . The exclusion criteria were outpatient status (27 patients), prior antibiotic usage (21 patients), inappropriate sputum (15 patients), did not attend the control visit on day 21 (7 patients), inability to perform spirometric tests (7 patients) and pneumonia (4 patients). Table 1 summarizes the patients' clinical characteristics.

PPMs recognized as agents causing respiratory infections, whether or not they belong to the oropharyngeal or gastrointestinal flora, were identified in $34(\% 45)$ of 75 patients (Table 2).

Tables 3 and 4 show serological results for $C h$. pneumoniae and M. pneumoniae.

There were mixed infections in 9 patients. In this mixed infection group, the most frequent micro-organisms were Ch. pneumoniae and H. influenzae (5 patients).

There was no statistical significance in terms of forced expiratory volume in one second $\left(\mathrm{FEV}_{1}\right)(\mathrm{p}=1)$, duration of hospital stay $(p=0.258)$, and requirement of noninvasive mechanical ventilation $(p=0.0689)$ between groups in which 
Table I Characteristics of the patients*

\begin{tabular}{|c|c|}
\hline No of subjects & 75 \\
\hline Male/female & $71 / 4$ \\
\hline Age & $61.1(41-77)$ \\
\hline \multicolumn{2}{|l|}{ Smoking } \\
\hline Smokers & $12(16 \%)$ \\
\hline Exsmokers & $59(78.6 \%)$ \\
\hline Nonsmokers & $4(5.35 \%)$ \\
\hline Pack-years & $43.6(6-100)$ \\
\hline Number of exacerbations last year & $2.3(0-6)$ \\
\hline Leukocytes $\left(\times 10^{9} / \mathrm{L}\right)$ & 10.127 \\
\hline \multicolumn{2}{|l|}{ Lung function } \\
\hline $\mathrm{FEV}_{1}, \mathrm{~L}$ & $0.774(0.4-1.63)$ \\
\hline $\mathrm{FEV}_{1}, \%$ pred & $30.8(13-59)$ \\
\hline FVC, L & $1.680(0.5 \mathrm{I}-2.4)$ \\
\hline FVC, \% pred & $42.5(20-86)$ \\
\hline $\mathrm{FEV}_{1} / \mathrm{FVC}$ & $50.2(35-7 \mathrm{I})$ \\
\hline \multicolumn{2}{|l|}{ Arterial blood gases } \\
\hline $\mathrm{PaO}_{2}(\mathrm{mmHg})$ & $58.9(37-75)$ \\
\hline $\mathrm{PaCO}_{2}(\mathrm{mmHg})$ & $43.2(12-72)$ \\
\hline $\mathrm{SatO}_{2}(\%)$ & $81.1(70-97)$ \\
\hline Patients using oxygen therapy & 70 \\
\hline Patients using NIMV & 40 \\
\hline
\end{tabular}

Note: *Values are given mean (\%) and mean (range).

Abbreviations: $\mathrm{FEV}_{1}$, forced expiratory volume in one second; FVC, forced vital capacity; NIMV, noninvasive mechanical ventilation.

an etiological agent responsible for acute exacerbation was identified or not. Bacteriological and serological results did not differ among patients using noninvasive mechanical ventilation (NIMV). According to the sensitivity results of PPMs cultivated from the patients, $H$. influenzae was resistant to penicilin in 10 patients, and to ampicillin in 9 patients (Table 5). In 4 patients $S$. pneumoniae was sensitive to almost all of the commonly used antibiotics.

\section{Discussion}

Although there is no widely accepted definition of acute exacerbation of COPD, either in clinical practice or research, a recent a consensus statement defined exacerbations as "a sustained worsening of the patient's condition, from the stable state and beyond normal day to day variations that is acute in onset and necessitates a change in regular medication in a patient with underlying COPD" (Rodriguez-Roisen 2000). Patients who suffer the most exacerbations have significantly lower health status (Seemungal et al 1998) and there is also some evidence that exacerbation frequency predicts an accelerated decline in lung function (Kanner et al 2001).
Infections play a major role in the etiology of acute exacerbations (Ball 1995). Patients with COPD have significant impairment of their lung defence mechanisms and colonisation of bronchial system seems to be an important consequence of the disease, both in stable state and in exacerbations. For more than 50 years, researchers have been trying to explain the meaning of bacteria recovered from a sputum culture in a COPD patient really means. Does the isolation of these bacteria along with mucus hypersecretion contribute to the pathogenesis of the disease? Is it simply a bacterial colonisation, or should it be treated? Distinguishing between colonisation and infection is difficult in these patients. In most of the reported series, the predominant organisms responsible for infective exacerbations are $S$. pneumoniae, nontypical H. influenzae, and to some extent Moraxella catarrhalis (Ball 1995; Monso et al 1995; Soler et al 1998; Miravitlles et al 1999). Our study results, in which the predominant bacteria isolated were $H$. influenzae, correlate with most of the previous ones in terms of bacterial etiology.

We have also investigated the role of Ch. pneumoniae and M. pneumoniae in acute exacerbations of COPD. We have seen an atypical etiology in 20 of 46 patients in whom an infectious etiology was determined from a total of 75 patients. This high percentage $(26 \%)$ implicates the role of atypical agents in the acute exacerbations of COPD. In 13 patients (17\%), the sole agent was Ch. pneumoniae or M. pneumoniae and there were mixed infections in 7 patients (9\%) with other bacteria. Data in the literature is uncertain on the role of atypical pathogens in the etiology of acute

Table 2 Microorganisms isolated from sputum cultures and serologic results for 75 patients

\begin{tabular}{ll}
\hline PPMs & \\
\hline Haemophilus influenzae' $^{\prime}$ & 23 \\
Streptococcus pneumoniae & 4 \\
Haemophilus parainfluenzae & 3 \\
Enterobacter aeruoginosa & $\mathrm{I}$ \\
Serratia marcescens & $\mathrm{I}$ \\
Klebsiella oxytoca & $\mathrm{I}$ \\
Klebsiella pneumoniae & $\mathrm{I}$ \\
Acinetobacter & $\mathrm{I}$ \\
Total PPM & 35 \\
Non-PPMs & 40 \\
Other & \\
Chlamydophila pneumoniae & \\
\multicolumn{1}{c}{ Mycoplasma pneumoniae } & 13 \\
\hline
\end{tabular}

Notes: Includes one mixed infection with S.Pneumoniae; ${ }^{2}$ Includes eight mixed infections; five with $H$. influenzae, two with $M$. pneumoniae and one with $K$. Pneumoniae. Abbreviation: PPMs, potentially pathogenic microorganisms. 
Table 3 Serologic results of Chlamydophila pneumoniae

\begin{tabular}{ll}
\hline Serology & Patient (number) \\
\hline $\lg M$ titer $>1: 16$ & $\mathrm{I}$ \\
$\lg G$ titer $>1: 512$ & $\mathrm{I}$ \\
A fourfold rise in $\lg \mathrm{G}$ titer & $\mathrm{II}$
\end{tabular}

exacerbations of COPD. Two studies of acute exacerbations (one in hospitalised patients and one in outpatients) found Ch. pneumoniae in approximately 5\% (Beaty et al 1991; Verkooyen et al 1997). Two other recent studies have found Ch. pneumoniae to be associated with $24 \%$ and $34 \%$ of exacerbations (Mogulkoc et al 1999; Karnak et al 2001). Because M. pneumoniae infects mainly young adults, and immunity may have been gained by the time acute exacerbation of COPD occurs, this agent may have a less important role in the etiology of advanced-age COPD patients. It is not surprising to see in most studies, as in ours, less evidence of exacerbations with M. pneumoniae than Ch. pneumoniae (Mogulkoc et al 1999; Lieberman et al 2001). We found only one study related to the role of Legionella species in acute exacerbation of COPD (Lieberman et al 2002). This study demonstrated serological evidence of infection with Legionella species in $16.7 \%$ of patients. We would have preferred to include this species in our study in addition to viral etiologies. But, technical and economic problems prevented us from studying these agents.

Functional impairment is a predictor of the bacteria responsible for acute infective exacerbations in COPD patients. In one study, more than $56 \%$ of patients with a $\mathrm{FEV}_{1} \leq 50 \%$ had Gram-negative-bacilli as etiology of acute exacerbation (Miravitlles et al 1999). Our study population had a mean $\mathrm{FEV}_{1}$ of $30 \%$, an even worse functional impairment, well correlated with this study with a high gramnegative predominance $(88 \%)$.

In this analysis of the sensitivity of micro-organisms to different antibiotics, we noted especially the poor efficacy of penicillin, ampicillin, amoxicillin-clavulanic acid, tetracyclin and erythromycin to most prevelant respiratory pathogens. Ciprofloxacin seems to be the most efficient drug for all microorganisms. The sensitivity results show severe functional impairment with Gram-negative predominance

Table 4 Serologic results of Mycoplasma pneumoniae

\begin{tabular}{ll}
\hline Serology & Patient (number) \\
\hline $\lg M>1: 16$ & $\mathrm{I}$ \\
$\lg G>1: 128$ & 3 \\
A fourfold rise in $\lg G$ titer & 3 \\
\hline
\end{tabular}

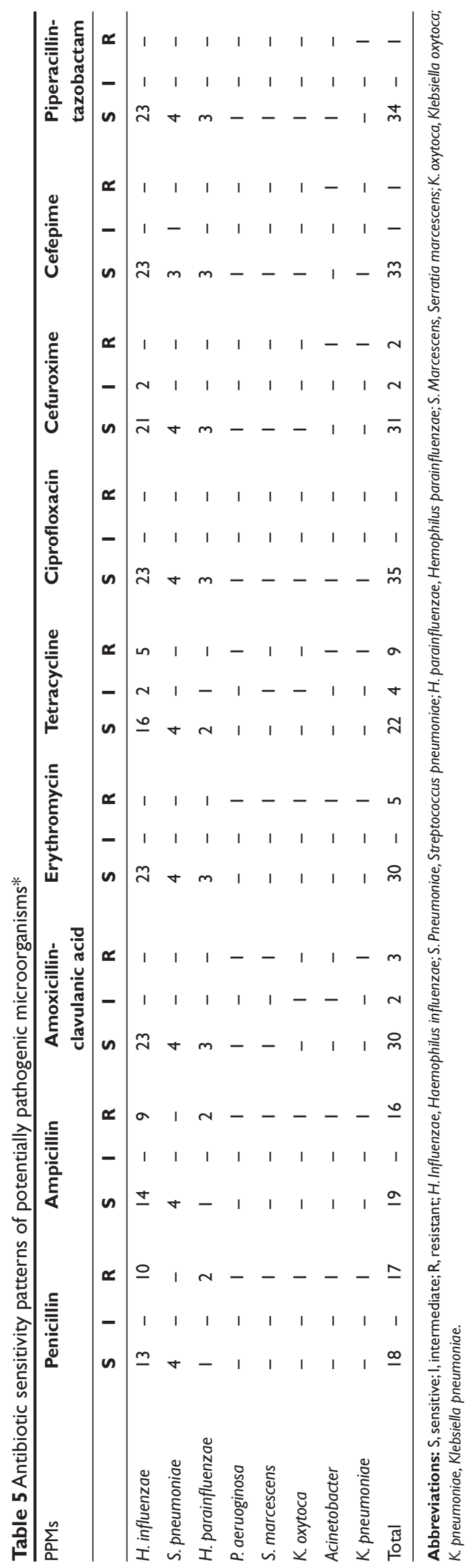


and also prior antibiotic usage during the lengthy course of COPD. These results were consistent with some studies in recent years (Miravitlles et al 1999), which showed similar sensitivity patterns in COPD patients.

In conclusion, atypical pathogens as well as typical bacteria may have a role in acute exacerbations of COPD. High resistance to commonly used antibiotics for typical agents and mixed infections in these severe functionally impaired cases of COPD were remarkable. We recommend that these results be considered when choosing an antibiotic in severe cases of COPD, although further studies are needed for clarification.

\section{Disclosure}

The authors report no conflicts of interest in this work.

\section{References}

[ATS] American Thoracic Society. 1995. Standards for the diagnosis, care of patients with chronic obstructive pulmonary disease. Am J Respir Crit Care Med, 152:S77-S120.

Ball P. 1995. Epidemiology and treatment of chronic bronchitis and its exacerbations. Chest, 108:43s-52s.

Beaty CD, Grayston JT, Wang SP, et al. 1991. Chlamydia pneumoniae, strain TWAR, infection in patients with chronic obstructive pulmonary disease. Am Rev Respir Dis, 144:1408-10.

Cabello H, Torres A, Celis R, et al. 1997. Bacterial colonization of distal airways in healthy subjects and chronic lung disease: a bronchoscopic study. Eur Respir J, 10:1137-44.

[GOLD] Global Initiative for Chronic Obstructive Lung Diseases. 2001. Global strategy for the diagnosis, management and prevention of chronic obstructive pulmonary disease: NHL/WHO workshop report, NIH publication No. 2701. Bethedsa: US Department of Health and Human Services.

Isenberg HD, Baron EJ, Damato RF, et al. 1991. Recommendations for isolation of bacteria from clinical specimens. In: Balows A, Hausler WJ Jr, Herrmann KL, et al. (eds). Manual of Clinical Microbiology. Washington, DC: American Society for Microbiology, pp. 216-37.
Kanner RE, Anthonisen NR, Connett JE. 2001. Lower respiratory illnesses promote FEV1 decline in current smokers but not ex-smokers with mild chronic obstructive pulmonary disease. Results from the lung health study. Am J Respir Crit Care Med, 164:358-64.

Karnak D, Beng Sun S, Beder S, et al. 2001. Chlamydia pneumoniae infection and acute exacerbation of chronic obstructive pulmonary disease (COPD). Respir Med, 95: 811-16.

Lieberman D, Lieberman D, Ben-Yaakov M, et al. 2001. Infectious etiologies in acute exacerbation of COPD. Diagn Microbiol Infect Dis, 40:95-102.

Lieberman D, Lieberman D, Ben-Yaakov M, et al. 2001. Chlamydia pneumonia infection in acute exacerbations of chronic obstructive pulmonary disease: Analysis of 250 hospitalizations. Eur J Clin Microbial Infect Dis, 20:698-704.

Lieberman D, Lieberman D, Shmarkov, et al. 2002. Serologic evidence of Legionella species infection in acute exacerbation of COPD. Eur Respir J, 19:392-7.

Miravitlles M, Espinosa C, Fernandez-Laso E, et al. 1999. Relationship between bacterial flora in sputum and functional impairment in patients with acute exacerbations of COPD. Chest, 116:40-6.

Mogulkoc N, Karakurt S, Isalska B, et al. 1999. Acute purulant exacerbations of chronic obstructive pulmonary disease and Chlamydia pneumonia infection. Am J Respir Crit Care Med, 160:349-53.

Monso E, Ruiz J, Rosell A, et al. 1995. Bacterial infection in chronic obstructive pulmonary disease. A study of stable and exacerbated outpatients using the protected specimen brush. Am J Respir Crit Care Med, 152:1316-20.

Murray PR, Washington JA. 1975. Microscopic and bacteriologic analysis of expoctrated sputum. Mayo Clin Proc, 50:339-44.

Rodriguez-Roisen R. 2000. Towards a consensus definition of COPD exacerbations. Chest, 117:398-401s.

Seemungal TA, Donaldson GC, Paul EA, et al. 1998. Effect of exacerbatin on quality of life in patients with chronic obstructive pulmonary disease Am J Respir Crit Care Med, 157:1418-22.

Soler N, Torres A, Ewig S, et al. 1998. Bronchial mcrobial patterns in severe exacerbations of chronic obstructive pulmonary disease (COPD) requiring mechanical ventilation. Am J Respir Crit Care Med, 157:1498-1505.

Verkooyen RP, Van Lent NA, Mousavi Joulandan SA, et al. 1997. Diagnosis of Chlamydia pneumoniae infection in patients chronic obstructive pulmonary disease by micro-immunofluorescence and ELISA. $J$ Med Microbiol, 46:959-64. 
